Supporting Information

\title{
Structural Properties and Hydrogen-Bonding Interactions in Binary Mixtures Containing a Deep-Eutectic Solvent and Acetonitrile
}

\author{
Payam Kalhor, Jing Xu, Hamad Ashraf, Bobo Cao, Zhi-Wu Yu*
}

MOE Key Laboratory of Bioorganic Phosphorous Chemistry and Chemical Biology, Department of Chemistry, Tsinghua University, Beijing 100084, China

A submission to The Journal of Physical Chemistry B

* Correspondence should be addressed to:

Prof. Zhi-Wu Yu

Tel.: (+86) 1062792492

Fax.: (+86) 1062771149

Email: yuzhw@tsinghua.edu.cn 

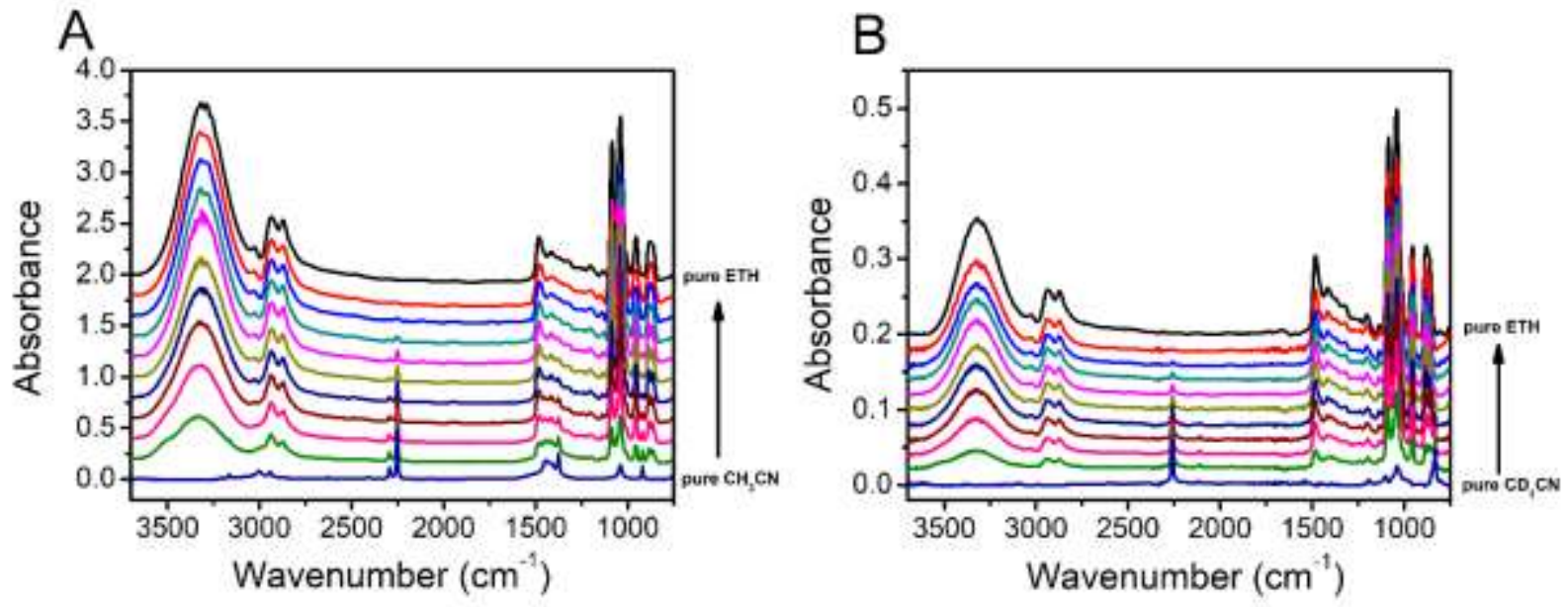

Figure S1. The FTIR spectra of $\mathrm{ETH}-\mathrm{CH}_{3} \mathrm{CN}(\mathrm{A})$ and $\mathrm{ETH}-\mathrm{CD}_{3} \mathrm{CN}(\mathrm{B})$ systems over the entire concentration range.

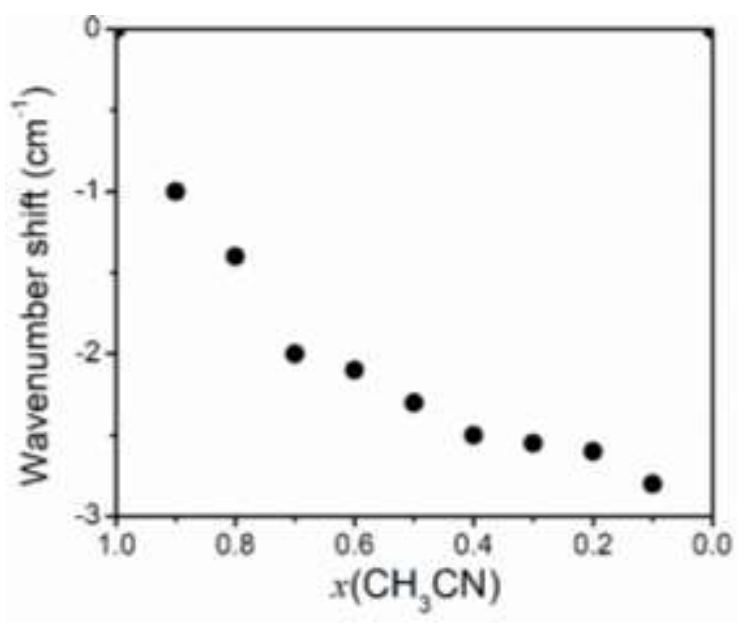

Figure S2. The wavenumber shift of $v(\mathrm{C} \equiv \mathrm{N})$ of IR real spectra for the system $\mathrm{ETH}-\mathrm{CH}_{3} \mathrm{CN}$.

Table S1. Calculated Bond Length and IR Stretching Frequency of $\mathrm{C} \equiv \mathrm{N}$ in the Complexes Shown in Figure 4 at M06-2X/6-311++G(d,p) Level of Theory ${ }^{\mathrm{a}}$

\begin{tabular}{cllcccc}
\hline complex & $\mathrm{r}_{\mathrm{C} \equiv \mathrm{N}}(\AA)$ & $v\left(\mathrm{~cm}^{-1}\right)$ & $\angle \mathrm{N} \ldots \mathrm{HBD}$ & $\mathrm{HBD}$ & $\mathrm{r}_{\mathrm{HB}}(\AA)$ & $\rho\left(\nabla^{2} \rho\right)(\mathrm{a} . \mathrm{u})$ \\
\hline $\mathrm{G}$ & 1.1478 & 2423.3 & - & - & - & - \\
$\mathrm{H}$ & 1.1488 & 2419.1 & 132.56 & $\mathrm{CH}$ & 2.48 & $0.0097(0.0322)$ \\
$\mathrm{K}$ & 1.1503 & 2401.7 & 150.05 & $\mathrm{OH}$ & 2.32 & $0.0108(0.0386)$ \\
$\mathrm{L}$ & 1.1496 & 2411.1 & 149.02 & $\mathrm{CH}$ & 2.48 & $0.0095(0.0306)$ \\
$\mathrm{M}$ & $1.1499\left(\mathrm{CH}_{3} \mathrm{CN}(1)\right)$ & 2405.6 & 165.44 & $\mathrm{OH}$ & 2.13 & $0.0159(0.0608)$ \\
& $1.1497\left(\mathrm{CH}_{3} \mathrm{CN}(2)\right)$ & 2404.4 & 152.84 & $\mathrm{CH}$ & 2.55 & $0.0082(0.0253)$ \\
$\mathrm{N}$ & $1.1487\left(\mathrm{CH}_{3} \mathrm{CN}(1)\right)$ & 2418.8 & 163.98 & $\mathrm{OH}$ & 1.98 & $0.0226(0.0847)$ \\
& $1.1496\left(\mathrm{CH}_{3} \mathrm{CN}(2)\right)$ & 2405.7 & 148.34 & $\mathrm{CH}$ & 2.49 & $0.0094(0.0301)$ \\
\hline
\end{tabular}

${ }^{\mathrm{a}} \mathrm{H}$-bond angle, length, electron density, and its Laplacian $\left(\nabla^{2} \rho\right)$ at BCP in different complexes are also given. 

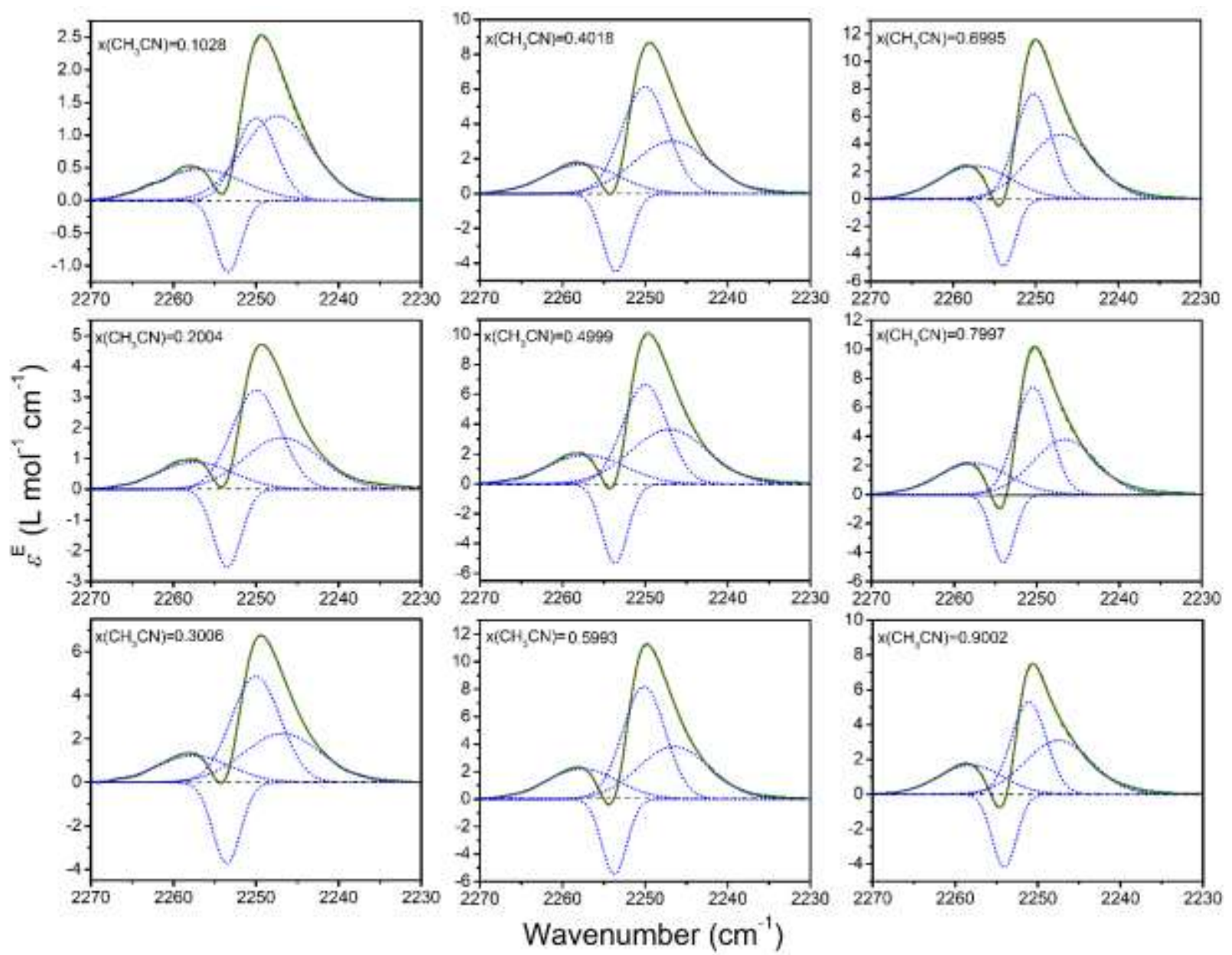

Figure S3. Deconvolution results of the excess IR spectra of the system $\mathrm{ETH}-\mathrm{CH}_{3} \mathrm{CN}$ in the region of $\mathrm{v}(\mathrm{C} \equiv \mathrm{N})$. 

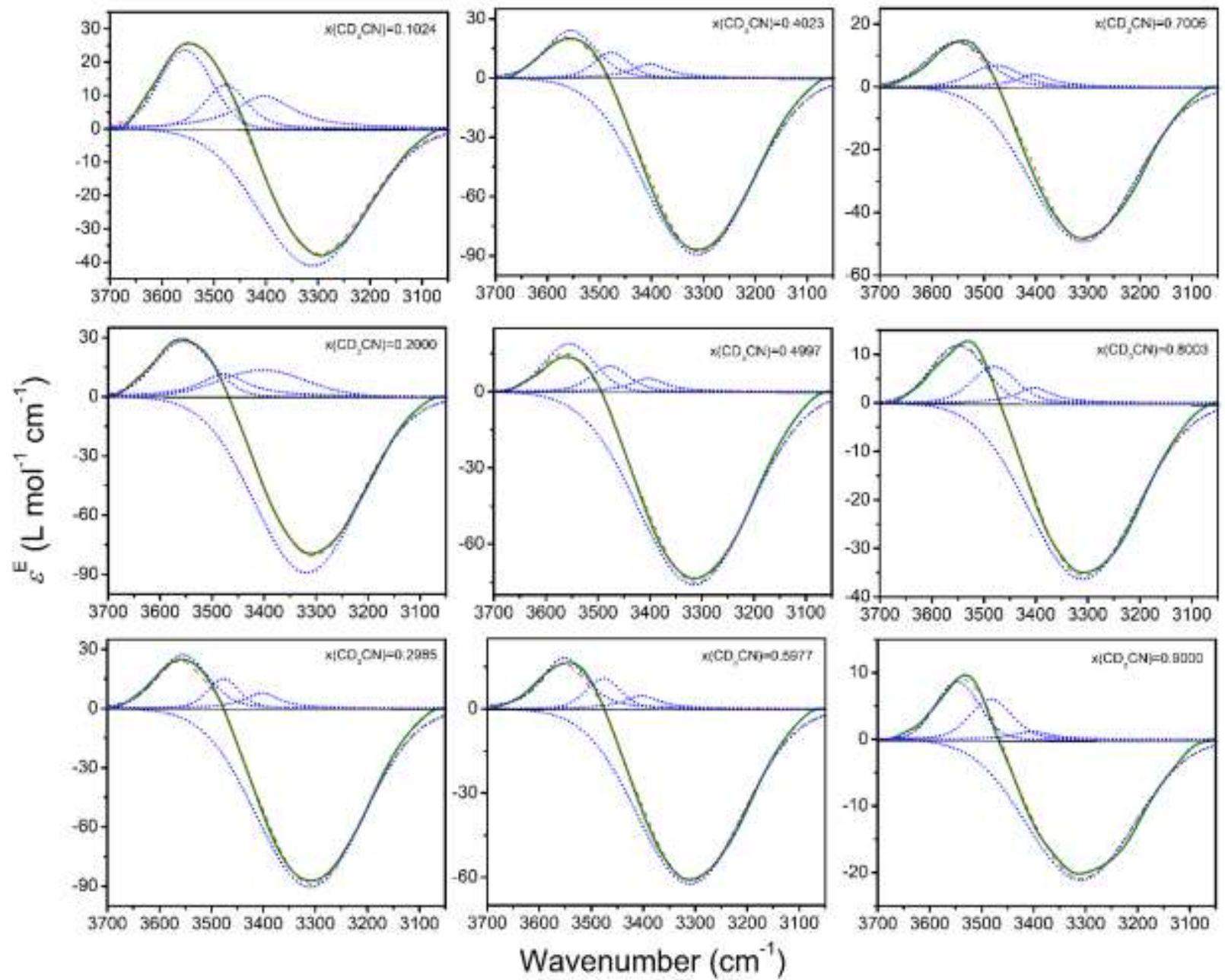

Figure S4. Deconvolution results of the excess IR spectra of the system $\mathrm{ETH}-\mathrm{CD}_{3} \mathrm{CN}$ in the region of $\mathrm{v}(\mathrm{O}-\mathrm{H})$. 
Table S2. Charges (q/e) on Selected Atoms and Molecules ${ }^{\text {a }}$

\begin{tabular}{|c|c|c|c|c|c|}
\hline atom/group & monomer & $\mathrm{ChCl}(\mathrm{A})$ & ETH (I) & $\mathrm{ETH}-1 \mathrm{CH}_{3} \mathrm{CN}(\mathrm{K})$ & $\mathrm{ETH}-2 \mathrm{CH}_{3} \mathrm{CN}(\mathrm{M})$ \\
\hline $\mathrm{Cl}^{-}$ & -1.000 & $\begin{array}{c}-0.889 \\
(0.111)\end{array}$ & $\begin{array}{c}-0.857 \\
(0.143)\end{array}$ & $\begin{array}{c}-0.859 \\
(0.141)\end{array}$ & $\begin{array}{c}-0.842 \\
(0.158)\end{array}$ \\
\hline $\mathrm{Ch}^{+}$ & 1.000 & $\begin{array}{c}0.889 \\
(-0.111)\end{array}$ & $\begin{array}{c}0.923 \\
(-0.077)\end{array}$ & $\begin{array}{c}0.931 \\
(-0.069)\end{array}$ & $\begin{array}{c}0.921 \\
(-0.079)\end{array}$ \\
\hline $3 \mathrm{CH}_{3}\left(\mathrm{Ch}^{+}\right)$ & 0.996 & $\begin{array}{c}0.930 \\
(-0.066)\end{array}$ & $\begin{array}{c}0.952 \\
(-0.043)\end{array}$ & $\begin{array}{c}0.958 \\
(-0.038)\end{array}$ & $\begin{array}{c}0.962 \\
(-0.034)\end{array}$ \\
\hline $2 \mathrm{CH}_{2}\left(\mathrm{Ch}^{+}\right)$ & 0.584 & $\begin{array}{c}0.555 \\
(-0.029) \\
\end{array}$ & $\begin{array}{c}0.566 \\
(-0.018) \\
\end{array}$ & $\begin{array}{c}0.568 \\
(-0.016) \\
\end{array}$ & $\begin{array}{c}0.571 \\
(-0.013)\end{array}$ \\
\hline $\mathrm{O}-\mathrm{H}\left(\mathrm{Ch}^{+}\right)$ & -0.236 & $\begin{array}{c}-0.245 \\
(-0.009)\end{array}$ & $\begin{array}{c}-0.248 \\
(-0.012)\end{array}$ & $\begin{array}{c}-0.247 \\
(-0.011)\end{array}$ & $\begin{array}{c}-0.267 \\
(-0.031)\end{array}$ \\
\hline $\mathrm{N}\left(\mathrm{Ch}^{+}\right)$ & -0.343 & $\begin{array}{c}-0.351 \\
(-0.008) \\
\end{array}$ & $\begin{array}{c}-0.347 \\
(-0.004) \\
\end{array}$ & $\begin{array}{c}-0.348 \\
(-0.005)\end{array}$ & $\begin{array}{c}-0.344 \\
(-0.001) \\
\end{array}$ \\
\hline $2 \mathrm{EG}$ & 0 & - & $\begin{array}{c}-0.066 \\
(-0.066)\end{array}$ & $\begin{array}{c}-0.068 \\
(-0.068)\end{array}$ & $\begin{array}{c}-0.081 \\
(-0.081)\end{array}$ \\
\hline CH2(EG1) & 0.268 & - & $\begin{array}{c}0.281 \\
(0.013)\end{array}$ & $\begin{array}{c}0.289 \\
(0.021)\end{array}$ & $\begin{array}{c}0.271 \\
(0.003) \\
\end{array}$ \\
\hline CH2(EG1) & 0.295 & - & $\begin{array}{c}0.279 \\
(-0.016)\end{array}$ & $\begin{array}{c}0.275 \\
(-0.02)\end{array}$ & $\begin{array}{c}0.281 \\
(-0.014)\end{array}$ \\
\hline $\mathrm{CH} 2(\mathrm{EG} 2)$ & 0.268 & - & $\begin{array}{c}0.286 \\
(0.018)\end{array}$ & $\begin{array}{c}0.288 \\
(0.020)\end{array}$ & $\begin{array}{c}0.290 \\
(0.022)\end{array}$ \\
\hline $\mathrm{CH} 2(\mathrm{EG} 2)$ & 0.295 & - & $\begin{array}{c}0.279 \\
(-0.016)\end{array}$ & $\begin{array}{c}0.280 \\
(-0.015)\end{array}$ & $\begin{array}{c}0.275 \\
(-0.020)\end{array}$ \\
\hline $\mathrm{CH}_{3} \mathrm{CN}(1)$ & 0 & - & - & $\begin{array}{c}-0.004 \\
(-0.004)\end{array}$ & $\begin{array}{c}0.002 \\
(0.002)\end{array}$ \\
\hline $\mathrm{C} \equiv \mathrm{N}(1)$ & -0.046 & - & - & $\begin{array}{c}-0.076 \\
(-0.030) \\
\end{array}$ & $\begin{array}{c}-0.069 \\
(-0.023) \\
\end{array}$ \\
\hline $\mathrm{CH}_{3}(1)$ & 0.046 & - & - & $\begin{array}{c}0.072 \\
(0.027) \\
\end{array}$ & $\begin{array}{c}0.071 \\
(0.025)\end{array}$ \\
\hline $\mathrm{CH}_{3} \mathrm{CN}(2)$ & 0 & - & - & - & $\begin{array}{c}0.000 \\
(0.000) \\
\end{array}$ \\
\hline $\mathrm{C} \equiv \mathrm{N}(2)$ & -0.046 & - & - & - & $\begin{array}{c}-0.070 \\
(-0.024)\end{array}$ \\
\hline $\mathrm{CH}_{3}(2)$ & 0.046 & - & - & - & $\begin{array}{c}0.070 \\
(0.024)\end{array}$ \\
\hline
\end{tabular}

a The data in the parentheses represent the charge changes $(\Delta \mathrm{q} / \mathrm{e})$ of the corresponding atoms or groups upon complexion. Positive and negative values mean decrease and increase in charge density, respectively 